\title{
Occupational Skin Diseases
}

Die beruflich bedingten Hauterkrankungen führen seit Jahren die Liste aller angezeigten Berufskrankheiten an und stellen neben dem medizinischen Problem auch ein großes sozialmedizinisches Problem dar, da sie hohe Kosten durch Arbeitsausfall, Arbeitsunfähigkeit und vor allem auch durch Umschulungen bei Berufswechsel verursachen. Hauterkrankungen liegen mit 53\% an erster Stelle, wenn man die Häufigkeitsverteilung der Berufskrankheiten analysiert.

Dennoch zeigt eine durch die Berufsgenossenschaften jährlich durchgeführte Statistik einen Rückgang der gemeldeten Berufskrankheiten. Während im Jahr 1997 noch 11247 Fälle gemeldet wurden, ging diese Zahl im Jahre 2001 auf 9815 Fälle zurück. Wesentlich für diesen Rückgang sind die Einleitung von professionellen Präventionsmaßnahmen, insbesondere für die Berufsbereiche der Friseure, Alten- und Krankenpfleger. Auch der Bereich der Latexallergie ist aufgrund der eingeleiteten Aufklärungsmaßnahmen sowie durch die Einführung der Technischen Regeln für Gefahrstoffe (TRGS 540) in den letzten Jahren zurückgegangen, einen Höchststand an gemeldeten Latexallergien gab es im Jahr 1998 mit 1262 Fällen, der sich im Jahre 2001 auf 392 reduzierte.

Die prozentualen Verteilungen berufsbedingter Hauterkrankungen innerhalb verschiedener Berufsgruppen weisen deutliche Unterschiede auf. An der Spitze stehen hier vor allem die Arbeitnehmer, die täglich mehr als 2 Stunden im feuchten Milieu arbeiten, sich häufig die Hände waschen oder mehr als 2 Stunden Schutzhandschuhe tragen müssen. Hier rangieren Friseure nach wie vor an der Spitze der berufsbedingten Hauterkrankungen, gefolgt von dem Bäckereigewerbe, wo neben Hauterkrankungen auch Atemwegserkrankungen ein Problem darstellen. Weitere wichtige Berufsbereiche sind die Metallberufe, Fliesenleger und die Gesundheitsberufe.
Das hier vorgelegte Themenheft „Berufsdermatologie“ umfasst Manuskripte zu aktuellen Themen der Prävention und Häufigkeit von berufsbedingten Hauterkrankungen sowie zu speziellen Fragestellungen wie der Latexallergie, Kontaktekzemen durch Kühlschmierstoffe und Untersuchungen zur Kontaktsensibilisierung gegenüber dem Duftstoffmix. Die Empfehlungen zur Begutachtung von Berufsdermatosen geben einen wichtigen Abschluss nach Darstellung der berufsbedingten Handekzeme im Nahrungsmittelgewerbe. Schließlich haben wir eigene Untersuchungen zur Penetration von Pollenallergenen durch die Haut eingebracht, da sie die Basis für die Entwicklung neuer Schutzsalben bilden. Insgesamt gibt das Heft einen sehr guten Überblick zur aktuellen Datenlage in dem Bereich der Berufsdermatologie, obgleich es aufgrund der limitierten Seitenzahl nicht möglich war, weitere wichtige Einzelbereiche, wie z.B. die Friseure oder Gärtner und Floristen, einzubeziehen.

Ich möchte mich bei allen Beteiligten, die an der Erstellung des Themenheftes aktiv mitgewirkt haben, herzlich bedanken und bin sicher, dass auch den Lesern diese interessante Zusammenstellung viel Freude beim Lesen bereitet. Ziel im Umgang mit Berufsdermatosen ist ihr Entstehen durch frühe Präventionsmaßnahmen zu verhindern bzw. wenn sie aufgetreten sind, durch optimale Maßnahmen ihrem Fortschreiten entgegenzuwirken. Die insbesondere durch die Berufsgenossenschaften eingeleiteten Maßnahmen und Aktivitäten zur Prävention von berufsbedingten Hauterkrankungen sind zu begrüßen und werden durch die Zusammenarbeit mit niedergelassenen Dermatologen sowie Schwerpunktzentren für berufsbedingte Hauterkrankungen tiefgreifend verbessert. Im Interesse der Arbeitnehmer ist diese interdisziplinäre Zusammenarbeit auch mit der Arbeitsmedizin weiter zu entwickeln und kontinuierlich zu verbessern. Viel Freude beim Lesen wünscht Ihnen

Ihre PD Dr. med. M. Worm 